\title{
A higher-order finite volume method with collocated grid arrangement for incompressible flows
}

\author{
L. Ramirez ${ }^{1}$, X. Nogueira ${ }^{1}$, S. Khelladi ${ }^{2}$, J. Chassaing $^{3}$ \& \\ I. Colominas ${ }^{1}$ \\ ${ }^{1}$ GMNI, Universidade da Coruña, Spain \\ ${ }^{2}$ Laboratoire de Dynamique des Fluides, Arts et Métiers ParisTech, \\ France \\ ${ }^{3}$ Institut Jean Le Rond d'Alembert, UPMC-CNRS, France
}

\begin{abstract}
A high accurate finite volume method based on the use of Moving Least Squares (MLS) approximants is presented for the resolution of the incompressible Navier-Stokes equations on unstructured grids. Moreover, in order to eliminate the decoupling between pressure and velocity, we present a new Momentum Interpolation Method that allows interpolations better than linear on any kind of mesh. The accuracy of the new method is evaluated by a steady and unsteady test cases.

Keywords: high-order methods, incompressible flow, Moving Least Squares, Momentum Interpolation Method, unstructured grids, collocated grids.
\end{abstract}

\section{Introduction}

Numerical solution of the Incompressible Navier-Stokes equations has a wide range of applications, such as low speed aerodynamics, biomedical fluid flow and hydrodynamics. In this kind of flows, the main problem with numerical solutions is to couple the changes of the velocity field with changes of the pressure field, and assuring at the same time that the continuity equation is satisfied. The staggered variable arrangement has been widely used for the discretization of the Incompressible Navier-Stokes equations, but it requires the use of structured meshes. However, most of engineering problems have a complex geometry making 
difficult the use of this kind of variable arrangement. On the other hand, in collocated grids, vector variables and scalar variables are stored at the same locations, usually in the centroid of the control volume. This seems to be a good approach to handle complex geometries. The main problem is the possibility of checker-board due to the central-difference discretization of the pressure.

In this context, the development of very accurate numerical methods to work on unstructured grids is very desirable. In this work we have proposed a new higher-order accurate finite volume formulation for the numerical solution of incompressible fluid flows on unstructured and collocated grids. The new formulation is based on the use of Moving Least Squares approximants. Moreover, in order to eliminate the decoupling between pressure and velocity, we present a new Momentum Interpolation Method that allows interpolations better than linear on any kind of mesh.

\section{Moving Least-Squares}

The Moving Least Squares (MLS) approach was originally devised in 1981 for data processing and surface generation [1]. The basic idea of the MLS approach is to approximate a function $u(\boldsymbol{x})$ at a given point $\boldsymbol{x}$ through a weighted least squares fitting on a compact domain around $\boldsymbol{x}$. The resulting approximation, $\widehat{u}(\boldsymbol{x})$, can be written as

$$
u(\boldsymbol{x}) \approx \widehat{u}(\boldsymbol{x})=\boldsymbol{N}^{T}(\boldsymbol{x}) \boldsymbol{u}_{\Omega_{\boldsymbol{x}}}=\boldsymbol{p}^{T}(\boldsymbol{x}) \boldsymbol{M}^{-1}(\boldsymbol{x}) \boldsymbol{P}_{\Omega_{\boldsymbol{x}}} \boldsymbol{W}(\boldsymbol{x}) \boldsymbol{u}_{\Omega_{\boldsymbol{x}}}
$$

where $N^{T}(x)$ is the vector of MLS shape functions and $\boldsymbol{u}_{\Omega_{\boldsymbol{x}}}$ contains the known values of the function $u(\boldsymbol{x})$ at the compact domain $\Omega_{\boldsymbol{x}}$. The vector $\boldsymbol{p}^{T}(\boldsymbol{x})$ represents an m-dimensional functional basis (polynomial in this work), $\boldsymbol{P}_{\Omega_{\boldsymbol{x}}}$ is defined as a matrix where the basis functions are evaluated at each point of the stencil and the moment matrix $\boldsymbol{M}(\boldsymbol{x})$ is obtained through the minimization of an error functional [2] and is defined as

$$
M(x)=P_{\Omega_{x}} W(x) P_{\Omega_{x}}^{T}
$$

The kernel or smoothing function, $W(\boldsymbol{x})$, plays an important role weighting the importance of the different points that take place in the approximation. A wide variety of kernel functions are described in the literature. In this work the exponential kernel has been used, defined in one dimension as

$$
W_{j}\left(x_{j}, x, s_{x}\right)=\frac{e^{-\left(\frac{d}{c}\right)^{2}}-e^{-\left(\frac{d_{m}}{c}\right)^{2}}}{1-e^{-\left(\frac{d_{m}}{c}\right)^{2}}}
$$

for $j=1, \ldots . ., n_{x}$, where $d=\left|x_{j}-x\right|, d_{m}=2 \max \left(\left|x_{j}-x\right|\right)$.

In equation (3) $d_{m}$ is the smoothing length, $n_{x}$ is the number of neighbors and $x$ is the reference point where the compact support is centered, the coefficient $c$ is defined by $c=\frac{d_{m}}{s_{x}}$ and $s_{x}$ is the shape parameter of the kernel. This parameter 
plays defines the kernel properties and therefore, the properties of the numerical scheme obtained.

As far as 2D applications are concerned, a multidimensional kernel can be obtained by means of the product of 1D kernels. For instance the $2 \mathrm{D}$ exponential kernel is defined by the following expression

$$
W_{j}\left(x_{j}, y_{j}, x, y, s_{x}, s_{y}\right)=W_{j}\left(x_{j}, x, s_{x}\right) W_{j}\left(y_{j}, y, s_{y}\right)
$$

The order of MLS approximations is determined by the polynomial basis used in the construction of MLS shape functions. Here we use quadratic and cubic polynomial basis. More details can be found in [3, 4].

The high order approximate derivatives of the field variables $u(\boldsymbol{x})$ can be expressed in terms of the derivatives of the MLS shape function. So the $n$th derivative is defined as

$$
\frac{\partial^{n} \widehat{u}}{\partial x^{n}}=\sum_{j=1}^{n_{x}} \frac{\partial^{n} N_{j}(\boldsymbol{x})}{\partial x^{n}} u_{j}
$$

We refer the interested reader to $[4,5]$ for a complete description of the computation of MLS derivatives.

\section{Governing equations and numerical discretization}

The Navier-Stokes equations in Cartesian coordinates can be written in nondimensional form as

$$
\begin{gathered}
\frac{\partial \boldsymbol{U}}{\partial t}+\boldsymbol{U} \cdot(\nabla \boldsymbol{U})=-\nabla p+\frac{1}{R e}(\Delta \boldsymbol{U}) \\
\nabla \cdot \boldsymbol{U}=0
\end{gathered}
$$

In this work we only address $2 \mathrm{D}$ problems, so $U=(u, v)^{T}$ is the velocity field. Moreover $p(x, y, t)$ is the pressure variable and $R e$ is the Reynolds number.

The continuity equation acts as a constraint in the velocity since the system of equations (6) and (7) is decoupled. Here we use the SIMPLE algorithm [6] to solve the system, and thus pressure is used as a mapping parameter. Figure 1 shows a flowchart of this algorithm. A number of inner iterations is required each time step to obtain a velocity field $U=(u, v)^{T}$ that satisfies equations (6) and (7). Every inner iteration the momentum equation (6) is used to predict the velocity field. A pressure correction equation is obtained from (6) and (7) to define the velocity and pressure corrections needed to satisfy the continuity equation (7). 


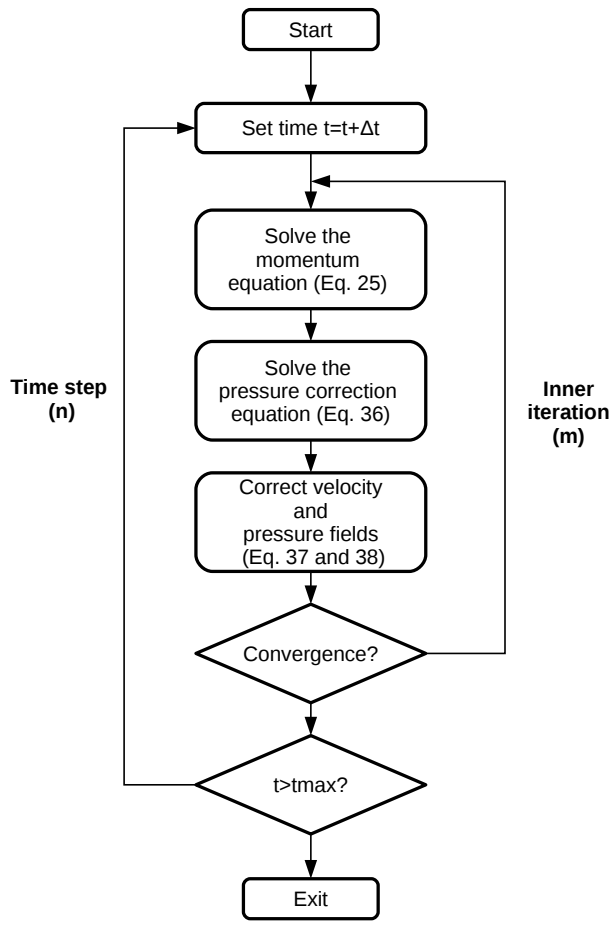

Figure 1: A flowchart for the unsteady SIMPLE algorithm.

A cell-centered finite volume scheme is applied to solve the incompressible Navier-Stokes equations (6)-(7). The discretized momentum equation reads as

$$
\begin{aligned}
& V_{I} \frac{3 \boldsymbol{U}_{I}^{m+1, n+1}-4 \boldsymbol{U}_{I}^{n}+\boldsymbol{U}_{I}^{n-1}}{2 \Delta t}+\sum_{j=1}^{N_{f}} \sum_{i g=1}^{N_{G}}\left[H_{j} \boldsymbol{U}_{j}\right]_{i g} \mathcal{W}_{i g} \\
& =-\sum_{j=1}^{N_{f}} \sum_{i g=1}^{N_{G}}\left[p_{j}^{m, n+1} \cdot \hat{\boldsymbol{n}}_{j}\right]_{i g} \mathcal{W}_{i g}+\frac{1}{R e} \sum_{j=1}^{N_{f}} \sum_{i g=1}^{N_{G}}\left[\nabla \boldsymbol{U}_{j}^{m+1, n+1} \cdot \hat{\boldsymbol{n}}_{j}\right]_{i g} \mathcal{W}_{i g}
\end{aligned}
$$

where $N_{f}$ is the number of faces of the control volume, $N_{G}$ represents the number of quadrature points, $\mathcal{W}_{i g}$ is the corresponding quadrature weight for the quadrature point at cell faces and $\hat{\boldsymbol{n}}_{j}$ is the unitary normal $\boldsymbol{n}$ multiplied by the area of the face $j$.

A deferred correction is used to obtain a high-order discretization of the convective term $U_{j}$ in equation (8). It reads as

$$
\boldsymbol{U}_{j}=\left(\boldsymbol{U}_{j}^{L O}\right)^{m+1, n+1}+\left(\boldsymbol{U}_{j}^{H O}-\boldsymbol{U}_{j}^{L O}\right)^{m, n+1}
$$


where the superscript $m, n+1$ refers to the previous inner iteration at the current time step $n+1$. In equation (9) the higher-order convective term, $U_{j}^{H O}$, is treated explicitly from the previous inner iteration $(m)$.

In this work we have approximated the terms $U_{j}^{H O}, p_{j}^{m, n+1}, \nabla U_{j}^{m+1, n+1}$ using Moving Least Squares approximants.

The term $H_{j}$ from equation (8) is written as

$$
H_{j}=\hat{\boldsymbol{U}}_{j} \cdot \hat{\boldsymbol{n}}_{j}
$$

where the value of $\hat{U}_{j}$ is obtained using the Momentum Interpolation Method (MIM) [7], as it will be explained later.

Once the velocity field is computed from equation (8) it is required to impose the continuity constraint given by equation (7). In order to satisfy this constraint the following pressure correction equation is solved

$$
\sum_{j=1}^{N_{f}} \sum_{i g=1}^{N_{G}}\left[\hat{\boldsymbol{U}}_{j} \cdot \hat{\boldsymbol{n}}_{j}\right]_{i g} \mathcal{W}_{i g}-\sum_{j=1}^{N_{f}} \sum_{i g=1}^{N_{G}}\left[\left(\frac{V_{I}}{a_{I}}\right)_{j}\left(\nabla p^{\prime}\right)_{j} \cdot \hat{\boldsymbol{n}}_{j}\right]_{i g} \mathcal{W}_{i g}=0
$$

where $a_{I}$ denotes the diagonal term of the mass matrix of the momentum equations for cell $I$. The terms $\left(\frac{V_{I}}{a_{I}}\right)_{j}$ and $\left(\nabla p^{\prime}\right)_{j}$ are obtained using MLS approximations. The value of $\hat{U}_{j}$ is computed using the Momentum Interpolation Method (MIM) as

$$
\hat{\boldsymbol{U}}_{j}=\boldsymbol{U}_{j}^{*}+\left(\frac{V_{I}}{a_{I}}\right)_{j}\left[\left(\overline{\nabla p_{I}}\right)_{j}-\nabla p_{j}\right]
$$

in equation (12) $U_{j}^{*}$ is the solution obtained with the momentum equations (8) approximated at integration points. We remark that all terms with the subscript $j$ of equation (12) require approximations since the known values are located at the centroid of the cells. Rhie and Chow method uses a linear interpolation to obtain the values of $\boldsymbol{U}^{*},\left(\frac{V_{I}}{a_{I}}\right),\left(\overline{\nabla p_{I}}\right)_{j}$ and $\nabla p_{j}$ at the integration point $j[7,8]$. We propose obtain these values by using higher-order MLS approximations as

$$
\begin{gathered}
\boldsymbol{U}_{j}^{*}=\sum_{k=1}^{n_{\boldsymbol{x}}} N_{k}\left(\boldsymbol{x}_{j}\right) \boldsymbol{U}_{k}^{*} \\
\left(\overline{\nabla p_{I}}\right)_{j}=\sum_{k=1}^{n_{\boldsymbol{x}}} N_{k}\left(\boldsymbol{x}_{j}\right) \nabla p_{k}
\end{gathered}
$$

In equation (14) the pressure gradient $\left(\nabla p_{k}\right)$ is obtained at the neighboring cell centroid $k$ as 


$$
\nabla p_{k}=\sum_{l=1}^{n_{\boldsymbol{x}}} \nabla N_{l}\left(\boldsymbol{x}_{k}\right) p_{l}
$$

Finally, the velocity and pressure fields are updated by

$$
\begin{gathered}
\boldsymbol{U}^{m+1, n+1}=\boldsymbol{U}^{*}+\boldsymbol{U}^{\prime}=\boldsymbol{U}^{*}-\frac{V_{I}}{a_{I}}\left(\nabla p^{\prime}\right)_{I} \\
p^{m+1, n+1}=p^{m, n+1}+\left(p^{\prime}\right)^{m+1, n+1}
\end{gathered}
$$

where $U^{*}$ is the velocity field obtained from the momentum equation (8). The superscript $m+1$ refers to the current inner iteration and $m$ refers to the previous inner iteration. In addition, $n+1$ refers to the current time iteration.

The resolution process is iteratively performed until velocity and pressure satisfy equations (6) and (7). We refer the interest reader to [3] for more details.

\section{Numerical results}

In this section we show two examples to test the accuracy of the new method.

\subsection{Kovasznay flow}

The first test case is the Kovasznay Flow [9]. This test case reproduces the laminar flow over a periodic array of cylinders. Since this flow introduces nonlinear effects it is a common benchmark to test the precision and the order of the numerical scheme $[10,11]$. The computational domain is defined as $\Omega=[-0.5,0.5] \times$ $[-0.5,0.5]$. The analytical solution has the form:

$$
\begin{array}{r}
u(x, y)=1-e^{\alpha x} \cos (2 \pi y), \quad v(x, y)=\frac{\alpha}{2 \pi} e^{\alpha x} \sin (2 \pi y) \\
p(x, y)=\frac{1}{2}\left(1-e^{2 \alpha x}\right)
\end{array}
$$

where the parameter $\alpha$ is given by $\alpha=\frac{R e}{2}-\sqrt{\frac{R e^{2}}{4}+4 \pi^{2}}$

Dirichlet boundary conditions are employed on all the boundaries where the values are prescribed with the analytical solution. The Reynolds number is 40 and different quadrilateral cell meshes of $32 \times 32,64 \times 64,128 \times 128$ and $256 \times 256$ are used in order to obtain the spatial order of convergence. A fourth order method is used to solve this problem.

Table 1 show the $L_{2}$ norm of the error in the variables $u, v$ and $p . L_{2}$ norm and the obtained order of accuracy. 
Table 1: Accuracy orders of velocity components and pressure field for Kovasznay flow test case with $R e=40$. A 4th order scheme is used. The expected order of convergence is recovered.

\begin{tabular}{|c|c|c|c|c|c|c|}
\hline & \multicolumn{2}{|c|}{$u$-velocity field } & \multicolumn{2}{c|}{$v$-velocity } & \multicolumn{2}{c|}{ Pressure } \\
\hline Mesh & $L_{2} u$ & Order $u$ & $L_{2} v$ & Order $v$ & $L_{2} p$ & Order $p$ \\
\hline \hline $32 \times 32$ & $1.41 \times 10^{-05}$ & - & $7.99 \times 10^{-06}$ & - & $2.64 \times 10^{-05}$ & - \\
\hline $64 \times 64$ & $1.00 \times 10^{-06}$ & 3.81 & $4.15 \times 10^{-07}$ & 4.27 & $1.20 \times 10^{-06}$ & 4.47 \\
\hline $128 \times 128$ & $6.55 \times 10^{-08}$ & 3.94 & $2.51 \times 10^{-08}$ & 4.05 & $6.96 \times 10^{-08}$ & 4.10 \\
\hline $256 \times 256$ & $4.17 \times 10^{-09}$ & 3.97 & $1.55 \times 10^{-09}$ & 4.02 & $4.98 \times 10^{-09}$ & 3.81 \\
\hline
\end{tabular}

\subsection{Viscous flow past a NACA 0015 airfoil}

In this section we compute the laminar flow around a NACA 0015 airfoil inside a channel at $R e=100$ and with an angle of attack of $\alpha=10$ degrees. The computational domain has a length of $2.2 c$ and a height of $0.41 c$, where $c$ denotes the chord of the airfoil.

The computational grid is shown in figure 2. It is an unstructured grid with 9469 quadrilateral cells.

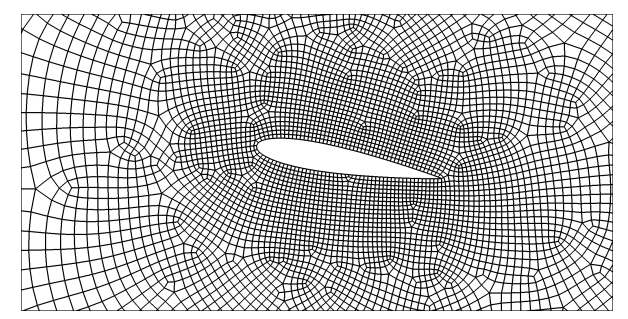

Figure 2: Unstructured mesh used for the computation of the laminar flow around the NACA 0015 airfoil. The mesh has 9469 quadrilateral cells.

The inflow velocity profile is given by $u(0, y)=\frac{4 U_{m} y(H-y)}{H^{2}}$ and $v(0, y)=0$, where $\mathrm{H}$ is the height of the domain and $U_{m}=1.5$ is the maximum velocity of the parabolic profile. Pressure outflow is prescribed at the outlet and no-slip boundary conditions are set on all remaining boundaries. The Reynolds number is defined by $R e=\bar{u} c / \nu$, where the value of the mean velocity is defined as $\bar{u}=\frac{2 U_{m}}{3}$. A third 
order version of the proposed method has been used for the computations. Results are plotted in figure 3 where we show the contours of velocity and pressure fields and contours.

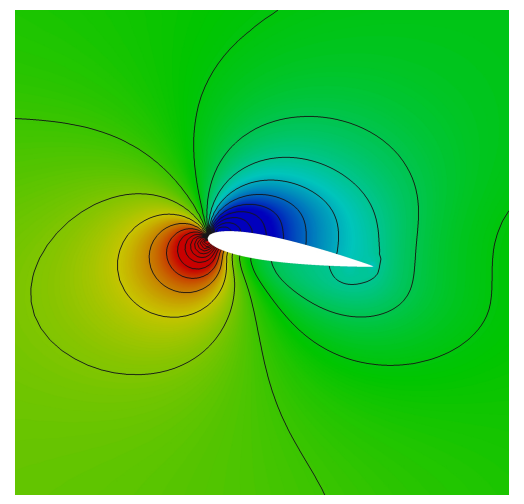

a)

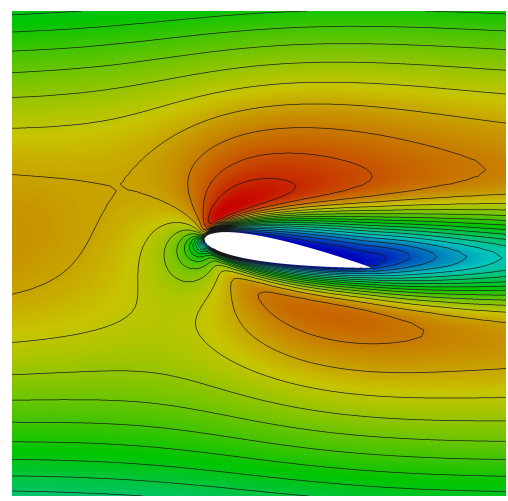

b)

Figure 3: Numerical results of the laminar flow around the NACA 0015 airfoil at $R e=100$. (a) The velocity field and contours and (b) the pressure field around the airfoil.

\section{Conclusions}

In this work we have presented a new methodology for the numerical solution of incompressible fluid flows on unstructured meshes based on the Moving Least Squares (MLS) approximations. It is a higher-order accurate finite volume formulation, and the SIMPLE algorithm is employed to impose iteratively the incompressibility condition. In order to avoid numerical instabilities a novel high order discretization of the Momentum Interpolation Method is proposed. The methodology presented is validated with some numerical examples, and we show that the formal order of accuracy is recovered. This novel formulation can be easily included in existing finite volume codes.

\section{References}

[1] Lancaster, P. \& Salkauskas, K., Pseudo-divergence-free element free galerkin method for incompressible fluid flow. Computer Methods in Applied Mechanics and Engineering, 193, pp. 1119-1136, 2004.

[2] Cueto-Felgueroso, L., Colominas, I., Nogueira, X., Navarrina, F. \& Casteleiro, M., Finite-volume solvers and moving least-squares approximations for the compressible Navier-Stokes equations on unstructured grids. Computer Methods in Applied Mechanics and Engineering, 196, pp. 4712 4736, 2007. 
[3] Ramirez, L., Nogueira, X., Khelladi, S., Chassaing, J. \& Colominas, I., A new higher-order finite volume method based on moving least squares for the resolutionof the incompressible Navier-Stokes equations on unstructured grids. Computer Methods in Applied Mechanics and Engineering, 278, pp. 883-901, 2014.

[4] Chassaing, J., Khelladi, S. \& X.Nogueira, Accuracy assesment of a highorder moving least squares finite volume method for compressible flows. Computers and Fluids, 71, pp. 41-53, 2013.

[5] Khelladi, S., Nogueira, X., Bakir, F. \& Colominas, I., Toward a higher-order unsteady finite volume solver based on reproducing kernel particle method. Computer Methods in Applied Mechanics and Engineering, 200, pp. 23482362, 2011.

[6] Patankar, S., Numerical heat transfer and fluid flow. Hemisphere Publishing Corporation, Taylor and Francis Group, 1980.

[7] Yu, B., Tao, W. \& Wei, J., Discussion on momentum interpolation method for collocated grids of incompressible flow. Numerical Heat Transfer, 42, pp. 141-166, 2002.

[8] Rhie, C. \& Chow, W., Numerical study of the turbulent flow past an airfoil with trailing edge separation. AIAA Journal, 21, pp. 1525-1532, 1983.

[9] Kovasznay, L., Laminar flow behind a two dimensional grid. Proc Cambridge Philos Soc, 44, pp. 58-62, 1948.

[10] Blasco, J., An anisotropic pressure-stabilized finite element method for incompressible flow problems. Computers and Mathematics with Applications, 53, pp. 895-909, 2007.

[11] Ranjan, R. \& Pantano, C., A collocated method for the incompressible Navier-Stokes equations inspired by the box scheme. Journal of Computational Physics, 232, pp. 346-386, 2013. 